\title{
THE EVOLUTIONARY STAGE OF H II REGION AND SPECTRAL TYPES OF MASSIVE STARS FROM KINEMATICS OF $\mathrm{H}_{2} \mathrm{O}$ MASERS IN W51 MAIN
}

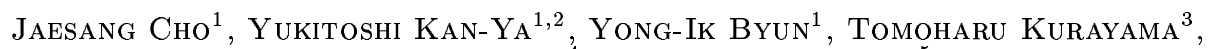 \\ YoON KYUNG $\mathrm{CHOI}^{4}$, AND Mi KYOUNG $\mathrm{KIM}^{5}$ \\ ${ }^{1}$ Department of Astronomy, Yonsei University, Shinchon-dong, Seodaemun-gu, Seoul 120-749, Korea \\ 2 KASI/KVN, Yonsei University, Shinchon-dong, Seodaemun-gu, Seoul 120-749, Korea \\ ${ }^{3}$ Faculty of Science and Engineering, Kagoshima University 1-21-35, Korimoto, Kagoshima, 890-0065, Japan \\ ${ }^{4}$ Max-Planck-Institut fuer Radioastronomie Auf dem Huegel 69, 53121 Bonn, Germany \\ ${ }^{5}$ Department of Astronomy, Graduate School of Science, The University of Tokyo, 7-3-1 Hongo, Bunkyo-ku, \\ Tokyo 113-0033, Japan
}

(Received January 28, 2010; Accepted March 30, 2010)

\begin{abstract}
We report relative proper motion measurements of $\mathrm{H}_{2} \mathrm{O}$ masers in massive star-forming region W51 Main, based on data sets of VLBI observations for $\mathrm{H}_{2} \mathrm{O}$ masers at $22 \mathrm{GHz}$ with Japanese VERA telescopes from 2003 to 2006 . Data reductions and single-beam imaging analysis are to measure internal kinematics of maser spots and eventually to estimate the three-dimensional kinematics of $\mathrm{H}_{2} \mathrm{O}$ masers in W51 Main. Average space motions and proper motion measurements of $\mathrm{H}_{2} \mathrm{O}$ masers are given both graphical and in table formats. We find in this study that W51 Main appears to be associated with hyper-compact H II region with multiple massive proto-stars whose spectral types are of late $\mathrm{O}$.
\end{abstract}

Key words : radio lines: stars — stars: formation — ISM: jets and outflows - ISM: kinematics masers: $\mathrm{H}_{2} \mathrm{O}$ maser

\section{INTRODUCTION}

The definition of massive stars is that they are OB stars sufficiently massive enough to produce type II supernovae with mass $>8 \mathrm{M}_{\odot}$ for solar abundance (Zinnecker \& Yorke 2007). Formation of massive stars is not well understood because of high dust extinctions in large distances and short time scale of evolutionary phases. However, it is very important to understand their evolutionary steps as they play a key role in the evolution of the Galactic abundance by the physical processes taking place in interstellar medium through supernova explosions and stellar winds. Understanding for triggers of massive star formation is based on the classical model of Elmegreen \& Lada (1977), in which OB stars are formed by gravitationally unstable layer between ionization of $\mathrm{H}$ II region and shock fronts on the molecular cloud complex (Fig. 1). In the local universe, formation of massive stars and star clusters are deeply linked because massive stars are found almost always in clusters (De Wit et al. 2005). H II regions and dust clouds surrounding clusters have been investigated by many researchers to understand clusters with massive stars. Thus, we also investigate the region where maser sources are found within shocked layers between ultra-compact $\mathrm{H}$ II (UCH II) region and the giant molecular cloud. About early-phase evolution, Beuther et al. (2007) proposed that high-mass star

Corresponding Author: J. Cho (b820120@galaxy.yonsei.ac.kr) forming cores have four evolutionary steps : High-mass starless cores (HMSCs) $\rightarrow$ High-mass cores harboring accreting low/intermediate mass protostar(s) destined to become high-mass star(s) $\rightarrow$ High-mass proto-stellar objects (HMPOs) $\rightarrow$ Finally massive stars. Hot molecular core (HMC) and hyper-compact H II (HCH II, $<0.01 \mathrm{pc}$ ) might coexist simultaneously. In massive star forming region, $\mathrm{HCH}$ II regions often consist of tight groups of two or more components (Sewilo et al. 2004) and are associated with strong $\mathrm{H}_{2} \mathrm{O}$ masers (Fig. 2 ). However, it is not clear yet whether which one is the former evolutionary stage between $\mathrm{HMC}$ and $\mathrm{HCH}$ II phase (Beuther et al. 2007). In these basic concepts about theory of the massive star formation, we can say that the maser source is one of the good probes to understand evolution of massive stars because their motions and kinematics are measurable.

$\mathrm{H}_{2} \mathrm{O}$ maser molecule was discovered toward several star-forming regions by Cheung et al. (1969) in the radio wavelength. $\mathrm{H}_{2} \mathrm{O}$ maser emissions with $\mathrm{OH}$ and $\mathrm{SiO}$ masers are important signposts for gas kinematics and magnetic field strength in massive star-forming region. When measuring with interferometry systems of high angular and velocity resolutions, maser emission can probe the star formation process, especially the earliest stage. The VLBI measurements indicate that the $\mathrm{H}_{2} \mathrm{O}$ masers are expanding away from the center in several massive star-forming regions. Torrelles et al. (1996) found that $\mathrm{H}_{2} \mathrm{O}$ masers trace both molecular outflows from newly-formed massive stars and accretion disks. 


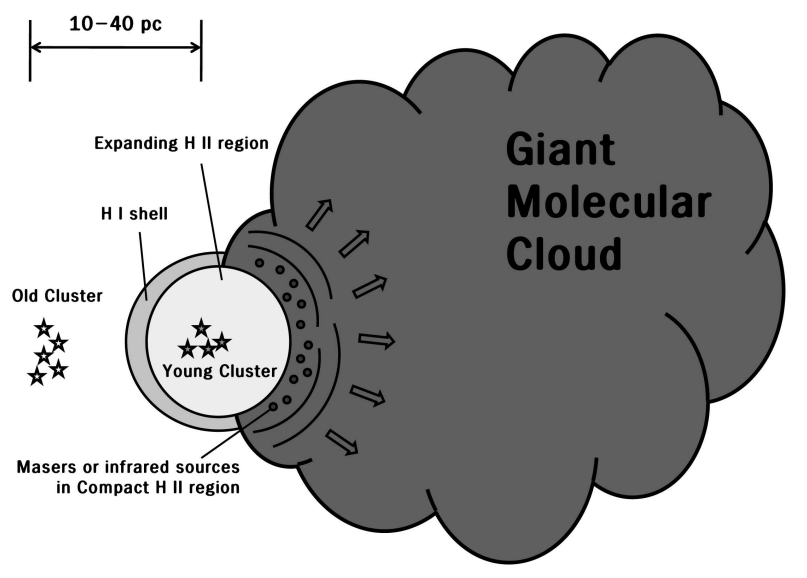

Fig. 1.- Schematic representation of massive star formation with expanding $\mathrm{H}$ II region toward giant molecular cloud (GMC) (Elmegreen \& Lada 1977).

Therefore, we can estimate the locations of young massive stars as originating points of the outflows and, in some cases, elucidate evolutionary status of the young stars and kinematics of the outflows themselves (Imai et al. 2002). The measurements of proper motions of many maser sources, together with the radial velocities and their spatial distribution, allow us to determine the three-dimensional kinematics (Genzel et al. 1981) and distance to the objects through parallax measurements. Based on estimated kinematics of maser spots and imaging of radio continuum sources, several investigators have postulated that $\mathrm{H}_{2} \mathrm{O}, \mathrm{CH}_{3} \mathrm{OH}$, and $\mathrm{OH}$ masers appear at different evolutionary stages of star formation. $\mathrm{H}_{2} \mathrm{O}$ masers seem to appear in the earliest stages of massive star formation during the period when the emerging protostar forms a detectable $\mathrm{UCH}$ II region.

There are about 1000 detected sources of $\mathrm{H}_{2} \mathrm{O}$ maser in the Galaxy. Among 420 sources associated with starforming regions, the Orion nebula is the massive starforming region studied most extensively because it is the closest region from the Sun. However, Orion nebula is not a rich site for the birth of $\mathrm{O}$ stars, therefore, investigations of more distant and luminous star-forming regions are needed. (Goldader \& Wynn-Williams 1994) The massive star-forming region W51 Main, our target source rich with $\mathrm{B}$ and $\mathrm{O}$ stars, contains numerous spots that emit strong $\mathrm{H}_{2} \mathrm{O}$ maser. These spots are likely to be associated with outflows from massive proto-stars. The W51 Main (as shown in Fig. 3) is a site of strong maser activity in about $30^{\prime \prime}$ east of W51 IRS 1 (the ellipse in the center of Fig. 3), and is defined by a group of $\mathrm{OH}$ and $\mathrm{H}_{2} \mathrm{O}$ masers (Genzel \& Downes 1977; Scott 1978) near some UCH II regions (Zhang \& Ho 1997). Genzel et al. (1981) used the method of statistical parallax of $\mathrm{H}_{2} \mathrm{O}$ masers around $\mathrm{UCH}$ II region W51 e2 (the ring under W51 Main in Fig. 3) (Scott 1978). The UCH II region near our tar-

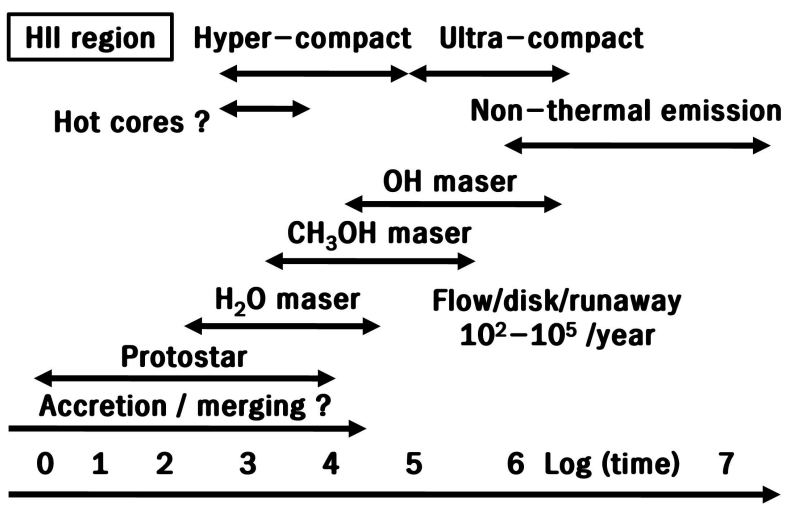

Fig. 2.- Possible chronology of non-thermal and maser emissions (Reid 2002; Imai 2006).

get source is classified with broad line recombination objects (BLROs) coupled with its small size and large internal motions (Jaffe \& Martin-Pintado 1999) and with shell-like morphology associated with molecular flows (Wood \& Churchwell 1989). Scott (1978) noted that spectral types of the stars are B0-09. There is no systematic motion, but random motions are found in the whole velocity ranges, so multiple outflows have been suspected (Imai et al. 2002), which means that there are probably multiple massive stars or protostellar condensations driving the maser emission. Observed proper motions of spots are affected by random shifts in the brightness maxima of individual maser spot, and also by Christmas tree effect due to appearance and disappearance of maser features during 20 years (Imai et al. 2002). For several reasons, as outlined in Genzel et al. (1981), the maximum lifespan of $\mathrm{H}_{2} \mathrm{O}$ maser is in the order of 10 years (Liljeström 1998; Leppänen et al. 1998). Emissions from W51 are almost $100 \%$ circularly polarized, and associated with strong magnetic fields of about 20-38 mG (Leppänen et al. 1998; Argon et al. 2002). Shocks would have affected or even triggered the star formation in W51 Main region (Leppänen et al. 1998) through gravitational contraction along the paralleled magnetic field lines to the minor axis of double cores, W51 e1 \& e2 (Lai et al. 2001).

This paper is structured as follows. Observational information and our method of the data analysis are described in section 2 . The results with measurements of proper motions for $\mathrm{H}_{2} \mathrm{O}$ maser features in W51 Main are presented in section 3 . Then, we will discuss about W51 Main as well as massive star formation in general in section 4. From these results and discussions, we finally make the conclusions with the mention of the future works and prospects in section 5 . 


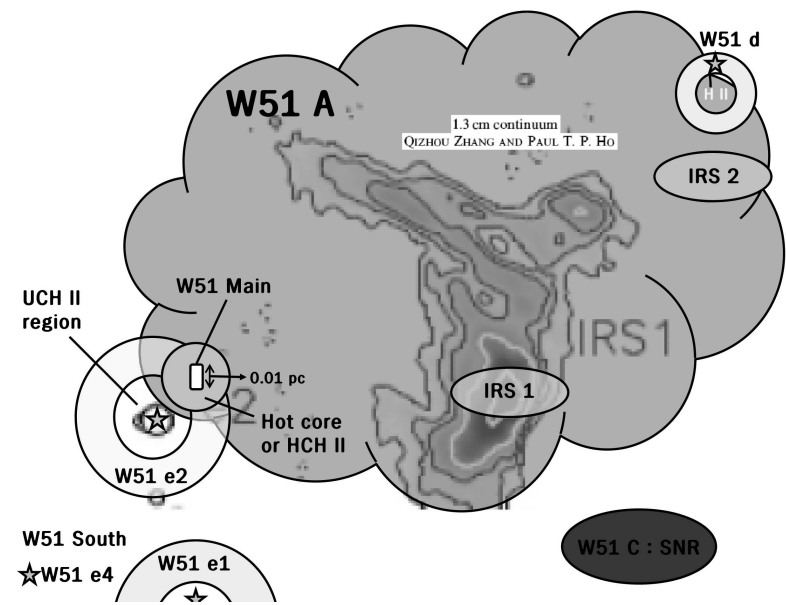

Fig. 3.- Schematic representation of W51 Main (the rectangle with $0.01 \mathrm{pc}$ scale) associated UCH II region, W51 e2 (the ring near W51 Main), with other objects in W51 A region. W51 $\mathrm{C}$ region is the supernova remnant also considered one of triggers for massive star formation in W51 A. Overlapped contour is from $1.3 \mathrm{~cm}$ emissions observed by Zhang \& Ho (1997). This figure is not in the scale of real relative size because it focuses on important factors near W51 Main.

\section{OBSERVATIONS AND DATA REDUC- TION}

\subsection{Observations}

The multi-epoch VLBI observations of $\mathrm{H}_{2} \mathrm{O}$ masers were performed with VERA at 10 epochs during the periods from 2003 to 2006 . The frequency of 22.235080 $\mathrm{GHz}\left(\mathrm{J}=6_{16} \rightarrow 5_{23}\right)$ was used for transitions of $\mathrm{H}_{2} \mathrm{O}$ masers in W51 Main. The interval of observations was about 2 months in 2004, but the other observations were not regular in 2003, 2005, and 2006. These 10 epochs are (year/day of year) : 2003/233, 2003/280, 2004/148, 2004/202, 2004/271, 2004/298, 2004/325, $2005 / 041,2005 / 132$, and 2006/031. At each epoch, the data were taken for 8 hours including calibrator observations by all stations. The spatial and radial velocity resolution of our observations are 1.2 milli-arcseconds (mas) and $0.42 \mathrm{~km} / \mathrm{s}$ corresponding frequency resolution of $31.25 \mathrm{kHz}$, respectively.

In our study, we determine relative position and motions of $\mathrm{H}_{2} \mathrm{O}$ masers in W51 Main region as a target source with the calibrator, J1924+1540. Both the target source and calibrator were observed simultaneously with dual beam system (Honma et al. 2003). The pointing position of W51 Main was $(\alpha, \delta)_{J 2000}=(19 \mathrm{~h}$ $\left.23 \mathrm{~m} 43 \mathrm{~s} .887,+14^{\circ} 30^{\prime} 36^{\prime \prime} .000\right)$. Dual beam mode requires a small separation, and the position of the calibrator is $(\alpha, \delta)_{J 2000}=\left(19 \mathrm{~h} 24 \mathrm{~m} 39 \mathrm{~s} .456,+15^{\circ} 40^{\prime}\right.$ $\left.43^{\prime \prime} .942\right)$.

For recording of the data received by left-handed circular polarization, the magnetic tapes are used at a rate of 1024 Mbps with the VERA DIR2000 recording

\section{Flow Chart of Data Reduction}

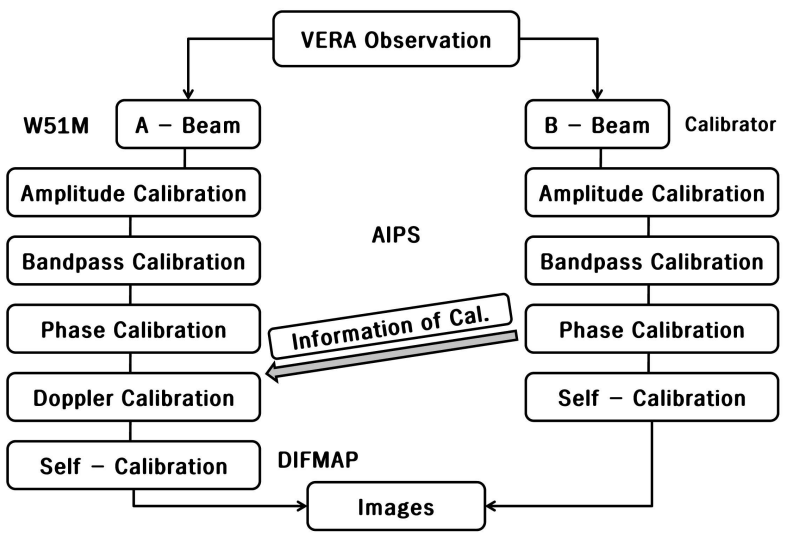

Fig. 4. - Flow chart of data reductions by using the AIPS and DIFMAP software package.

system, providing a total bandwidth of $256 \mathrm{MHz}$ with 2-bit quantization, and filtered by the VERA digital filter unit (Iguchi et al. 2005). The $256 \mathrm{MHz}$ bandwidth data were split into 16 IFs each with $16 \mathrm{MHz}$ bandwidth. For the continuum source J1924+1540, 15 IFs are used with gaps between IFs of $250 \mathrm{MHz}$, while one IF for W51 Main (corresponding frequency in the 8 th IF for the calibrator) was divided into 512 spectral channels for $\mathrm{H}_{2} \mathrm{O}$ masers in W51 Main. The data were correlated with integration time of 1 second by the Mitaka FX correlator (Chikada et al. 1991) located at the NAOJ Mitaka campus. The system temperature including atmospheric attenuation were measured with the chopper-wheel method (Ulich \& Hass 1976) to be typically $100-500 \mathrm{~K}$, depending on the weather condition and elevation angle of the sources. The aperture efficiencies of the antennas ranged from 45 to $52 \%$, depending on the stations.

\subsection{Data Reductions}

We have performed data reductions with the AIPS (Astronomical Image Processing Software) tool of the NRAO (National Radio Astronomy Observatory), and mapping W51 Main region with DIFMAP package. We have done reductions of single-beam analysis for data of an epoch in 2006 and combined our result with singlebeam imaging results of Imai et al. (2005) and Kanya et al. (2007) from which they calculated proper motions of maser spots from 2003 to 2005.

We carried out single-beam VLBI imaging processes for the calibrator, J1924+1540. The tables with information after calibrations for J1924+1540, the solution tables, were applied to one of the reduction processes for W51 Main when we made phase calibrations for the A-beam. During the process of reductions for W51 Main, we selected a maser spot (R) in the 160th channel after the doppler calibrations, having almost constant value of phase delays, as a reference maser (R) 
to perform the CLEAN (synthesis imaging and deconvolution) process allocating a CLEAN box to map by DIFMAP.

Fig. 4 shows the flow chart of our reduction processes. The amplitude calibrations were made from system noise temperature (task ACCOR and APCAL in AIPS). Bandpass calibrations were performed using auto-correlation spectra of continuum sources (task BPASS). For phase calibrations, the visibilities of all velocity channels were referenced to the reference maser spot, one of the brightest sources, by using the continuum source with delays, and the line source, W51 Main, without delays (task FRING). Doppler calibrations converted the center (the 256th channel) of bandpass to the radial velocity with respect to the Local Standard of Rest (LSR) velocity, $\mathrm{V}_{L S R}=65.4 \mathrm{~km} / \mathrm{s}$ (Fomalont \& Weliachew 1973), of W51 and the rest frequency of $22.235080 \mathrm{GHz}$ of $\mathrm{H}_{2} \mathrm{O}$ masers (task CVEL). Self-calibrations were made for the reference spot using DIFMAP software package. The image size of each map and channel was 204.8 and 409.6 mas with a pixel size of 0.1 mas $\times 0.1$ mas.

After all calibrations, radial velocity mapping is also done by maser spots from a number of channels from the 195 th to the 320 th channel, covering the LSR velocity range between $38.44 \mathrm{~km} / \mathrm{s}$ and $133.22 \mathrm{~km} / \mathrm{s}$, expected for these masers on DIFMAP. In order to identify the maser spot in each channel, we used a set of criteria, which are (1) the maser should exist in the same position through at least two channels, (2) the peak value of the flux of the maser should be larger than seven times deviation value of rms, and (3) the maser should have side-lobe in the map. Based on these criteria, we measured positions with errors of maser spots by fitting elliptical Gaussian brightness distribution to the image with the AIPS task JMFIT. The resultant synthesized beam size (FWMH) was typically 1.53 mas $\times 0.75$ mas with a position angle of about -33 degree. The formal uncertainty of the maser positions given by DIFMAP was better than about 0.05 mas.

For the analysis of multi-epoch VLBI data, we need to identify the same maser spot in different epochs based on positions as well as radial velocities of spots. These spots were identified as the same if their positions were within 1 mas and their radial velocities were within $0.84 \mathrm{~km} / \mathrm{s}$, which is twice the value of velocity resolution. Their proper motions were then determined based on a linear least-square fitting, for at least three epoch detections more than three epochs (Honma et al. 2005; Sato et al. 2007).

\section{RESEULTS}

\subsection{Measurements of Proper Motions}

Proper motion of a maser is useful to understand its environments and the velocity fields around massive (proto-) stars. We show the results from single-beam data analysis to see the relative positions and internal motions of $\mathrm{H}_{2} \mathrm{O}$ masers in W51 Main region. Based on the criteria described in section 2.2 , we have identified 62 maser spots (see Appendix A). In principle, we should find a maser that shows up in all epochs so that we can compare with the positional reference maser $(\mathrm{R})$ of the epoch in 2006. However, there was no such maser and therefore, we divided observation epochs into three groups - the 1st group : the 1 st-4th, the 2 nd group : the 4 th- 8 th, and the 3rd group : the 8 th-10th - in the order of the time sequences of ten epoch data to fit the origin of each map center. Each group contains at least one overlapping maser because all maps should be shifted again later between the maps to the center of the origin of the 10th epoch (2006/031), which we have analyzed with the reference maser $(\mathrm{R})$. We set reference spots in each group, and then all of the proper motion vectors relative to the proper motion of the reference spot are calculated. When we selected the reference masers, we have considered that the masers should have high radial velocities for the smallest bias to the proper motions of other maser spots. We note that Genzel et al. (1981) claim that space velocities of maser features can be similar in spite of big differences of radial velocities.

Now, all positions of maser spots are relative to the proper motion of the reference spot itself. We subtracted mean value of proper motion velocities for 62 maser features to proper motions of others to consider systematic motions of reference frame (cf. Honma et al. 2005). There might occur a bias to the proper motions of other features if reference maser spot itself has a large proper motion. However, the average value of $\mu_{X}$ and $\mu_{Y}$ for 62 features are $-0.022 \mathrm{mas} / \mathrm{yr}$ and $-0.059 \mathrm{mas} / \mathrm{yr}$, respectively, corresponding to -1.821 $\mathrm{km} / \mathrm{s}$ and $-2.698 \mathrm{~km} / \mathrm{s}$ at a source distance of $8.5 \mathrm{kpc}$. We can say that these values are not large compared to radial velocity width on the order of a few tens in $\mathrm{km} / \mathrm{s}$ from their spectra. Therefore, reference maser spot (R) does not make a large systematic motions of reference frame as we expected.

\subsection{Proper motions in W51 Main}

Table 1 summarizes the results of proper motion measurements, obtained by linear least-square-fitting method, as well as LSR velocities, positions, detected epochs, RMS residuals, and total proper motion velocities for all of our maser samples. The proper motion vectors listed in Table 1 are illustrated in Fig. 5. Among them, a maser feature, ID 2, has the longest lifespan in our data from the 1st to 9th epoch corresponding to around 629 days. Moreover, we could find Christmas tree effect in some features as mentioned in previous studies (Genzel et al. 1981; Imai et al. 2002). 
Table 1.

Proper motion measurements from 2003 to 2006

\begin{tabular}{|c|c|c|c|c|c|c|c|c|c|}
\hline $\mathrm{ID}^{1}$ & $\mathrm{~V}_{L S R}{ }^{2}$ & $\mathrm{X}^{3}$ & $\mathrm{Y}^{4}$ & $\mu_{X}{ }^{5}$ & $\mu_{Y}{ }^{6}$ & Epochs ${ }^{7}$ & $\sigma_{X}^{8}$ & $\sigma_{Y}^{9}$ & $\mathrm{~V}_{P}{ }^{10}$ \\
\hline 1 & 100.10 & -6.631 & -12.438 & -1.317 & 0.618 & 1110000000 & 0.222 & 0.115 & 25.41 \\
\hline 2 & 97.87 & -0.354 & -17.920 & 0.034 & 2.577 & 1101111110 & & 0.021 & 104.22 \\
\hline 3 & 95.10 & -0.020 & -15.551 & -0.763 & 2.196 & 1111000000 & & 0.068 & 91.81 \\
\hline 4 & 95.31 & 6.626 & -9.370 & -0.708 & 1.918 & 1111010000 & 0.094 & 0.033 & \\
\hline 5 & 89.00 & 23.864 & -3.700 & 1.548 & -1.206 & 1111110000 & 0.037 & 0.019 & 78.69 \\
\hline 6 & 52.72 & -59.285 & -78.213 & 4.221 & -2.062 & & & & 189.79 \\
\hline 7 & 47.61 & -57.845 & -76.196 & -0.626 & -3.222 & 0001111000 & 0.030 & & 130.61 \\
\hline 8 & 122.65 & -6.970 & -48.414 & -1.976 & -1.331 & & 0.469 & & 54.24 \\
\hline 9 & 117.17 & -49.485 & 9.142 & 1.796 & & & & & \\
\hline 10 & & -39.857 & -8.611 & & & & & & 154.58 \\
\hline 11 & & 0. & & 11.1 & & & & & \\
\hline 12 & 1. & -49.2 & 8.087 & & & & & & \\
\hline 13 & & & -4.328 & & & & & & 52.55 \\
\hline 14 & & -4.8 & -4.9 & -0.9 & & & & & \\
\hline 15 & 3.98 & -22.7 & -4.032 & -0.1 & -1.186 & & 0.1 & & 47.55 \\
\hline 16 & 82.61 & -52.484 & -70.520 & & -0.045 & & & & 5.18 \\
\hline 17 & & & & & & & & & \\
\hline 18 & & & & & & & & & \\
\hline 19 & 65.86 & -24.6 & 74.067 & 1.2 & -3.920 & & 0.330 & & 162.35 \\
\hline 20 & & -71 . & & & & & 0.023 & & 105.84 \\
\hline $21^{R}$ & & & -32.638 & & & & & & 3.31 \\
\hline 22 & & -3.094 & & -1.3 & -4.073 & & & & 172.85 \\
\hline 23 & 4.96 & -2.614 & -4.468 & 3.892 & -2.568 & & & & 185.49 \\
\hline 24 & & & & & & & & & \\
\hline 25 & & & & & & & & & \\
\hline 26 & & 8.488 & & -1.038 & -1.699 & & & & 78.53 \\
\hline 27 & & & & & & & & & \\
\hline 28 & & & & & & & & & .18 \\
\hline 29 & & -26.52 & & -0.1 & & & & & 4.02 \\
\hline 30 & & & & & & & & & 1.52 \\
\hline 31 & & & & & & & & & 2.97 \\
\hline 32 & & & & & & & & & 35.24 \\
\hline 33 & & & & & & & & & \\
\hline 34 & & & & & & & & & 28.59 \\
\hline 35 & & & & & & & & & 41.50 \\
\hline 36 & & & & & & & & & 34.67 \\
\hline 37 & & & & & & & & & \\
\hline 38 & & & & & & & & & 49.24 \\
\hline 39 & & -17.8 & 50.8 & & & & & & 90.17 \\
\hline 40 & & & & & & & & & \\
\hline 41 & & & & & & & & & 55.30 \\
\hline 42 & & -24.106 & & -0.3 & -0.327 & & & & 17.80 \\
\hline 43 & & & & & & & & & \\
\hline 44 & & & & & & & & & \\
\hline 45 & 51.48 & -56.475 & -75.052 & -1.228 & -1.238 & 1110000000 & 0.012 & 0.409 & 52.89 \\
\hline
\end{tabular}

Note : 1 . Spot ID numbers; 2 . LSR velocities in $\mathrm{km} / \mathrm{s}$ averaged over detected epochs; 3,4 . Best-fit offset positions [R.A., Dec.] at the first epoch in mas; 5, 6. Best-fit proper motions in mas/yr; 7. Detected epochs : 1 for detection, and 0 for non-detection; 8, 9. RMS position residuals from the best-fit proper motions in mas; and 10. Total proper motion velocities of the maser spots in $\mathrm{km} / \mathrm{s} ; \mathrm{R}$ : Reference maser source. 
Table 1 (Continued).

Proper motion measurements from 2003 to 2006

\begin{tabular}{rrrrrrrrrr}
\hline \hline & & & & & & & & & \\
$\mathrm{ID}^{1}$ & $\mathrm{~V}_{L S R}{ }^{2}$ & $\mathrm{X}^{3}$ & $\mathrm{Y}^{4}$ & $\mu_{X}{ }^{5}$ & $\mu_{Y}{ }^{6}$ & Epochs & $\sigma_{X}{ }^{8}$ & $\sigma_{Y}{ }^{9}$ & $\mathrm{~V}_{P}{ }^{10}$ \\
\hline 46 & 111.06 & -44.085 & -58.352 & -2.641 & 2.443 & 0011100000 & 0.145 & 0.817 & 129.35 \\
47 & 97.33 & -54.414 & -35.559 & 0.022 & 0.062 & 0011111100 & 0.001 & 0.117 & 3.39 \\
48 & 96.68 & -32.949 & 14.238 & 1.432 & 0.817 & 0011010110 & 0.041 & 0.003 & 65.57 \\
49 & 88.53 & -22.165 & -4.633 & -2.565 & -2.272 & 0011010000 & 0.300 & 0.152 & 134.83 \\
50 & 79.83 & -18.215 & 48.517 & 0.931 & 5.002 & 0011100000 & 0.406 & 0.402 & 203.76 \\
51 & 71.73 & -21.942 & 89.440 & -1.086 & -1.388 & 0111000000 & 0.148 & 0.358 & 56.89 \\
52 & 60.07 & -29.314 & 74.483 & -0.508 & -0.993 & 0111100000 & 0.068 & 0.168 & 42.87 \\
53 & 57.84 & -30.305 & 73.037 & -0.772 & -2.153 & 0011100000 & 0.286 & 0.702 & 86.07 \\
54 & 56.60 & -55.955 & 41.747 & -0.679 & -3.222 & 0011100000 & 0.023 & 0.346 & 131.02 \\
55 & 55.88 & -84.985 & -27.143 & -1.264 & -2.147 & 0011010000 & 0.283 & 0.362 & 87.36 \\
56 & 50.05 & -58.435 & -77.913 & -0.441 & -1.688 & 0011100000 & 0.052 & 1.031 & 67.33 \\
57 & 113.76 & -8.335 & -10.573 & -2.430 & -2.693 & 0001110100 & 0.223 & 0.105 & 110.94 \\
58 & 107.35 & -0.725 & -3.623 & 1.185 & -0.171 & 0001110000 & 0.753 & 0.228 & 25.63 \\
59 & 107.74 & 1.975 & -3.663 & -0.940 & 1.071 & 0001110000 & 0.797 & 0.232 & 43.76 \\
60 & 98.05 & 13.505 & 5.317 & -0.789 & 0.991 & 0001110000 & 0.974 & 0.110 & 133.39 \\
61 & 106.31 & -8.455 & -11.703 & -5.034 & 2.881 & 0001111000 & 0.352 & 0.187 & 50.68 \\
62 & 82.58 & 85.921 & -60.444 & -0.092 & 0.441 & 0001110000 & 1.997 & 1.042 & 18.30 \\
\hline
\end{tabular}

Note : 1. Spot ID numbers; 2. LSR velocities in $\mathrm{km} / \mathrm{s}$ averaged over detected epochs; 3,4 . Best-fit offset positions [R.A., Dec.] at the first epoch in mas; 5, 6. Best-fit proper motions in mas/yr; 7. Detected epochs : 1 for detection, and 0 for non-detection; 8, 9. RMS position residuals from the best-fit proper motions in mas; and 10. Total proper motion velocities of the maser spots in $\mathrm{km} / \mathrm{s}$.

Table 2.

Space motion measurements from 2003 to 2006

\begin{tabular}{crrrrr}
\hline \hline & & & & & \\
ID & $\mathrm{V}_{L S R}[\mathrm{~km} / \mathrm{s}]$ & $\mathrm{V}_{X}[\mathrm{~km} / \mathrm{s}]$ & $\mathrm{V}_{Y}[\mathrm{~km} / \mathrm{s}]$ & $\mathrm{V}_{Z}{ }^{*}[\mathrm{~km} / \mathrm{s}]$ & $\mathrm{V}_{S}[\mathrm{~km} / \mathrm{s}]$ \\
\hline 1 & & & & & \\
2 & 100.10 & -22.01 & 12.70 & 34.70 & 43.01 \\
3 & 97.87 & 2.34 & 104.20 & 32.47 & 109.16 \\
4 & 95.10 & -29.13 & 87.07 & 29.70 & 96.50 \\
5 & 95.31 & -25.55 & 72.49 & 29.91 & 82.47 \\
6 & 89.00 & 62.63 & -47.65 & 23.60 & 82.16 \\
7 & 52.72 & 170.85 & -82.64 & -12.68 & 190.21 \\
8 & 47.61 & -24.06 & -128.38 & -17.79 & 131.82 \\
9 & 122.65 & -45.20 & -29.98 & 57.25 & 78.86 \\
10 & 117.17 & 71.62 & -122.56 & 51.77 & 151.10 \\
11 & 116.81 & 46.58 & 147.40 & 51.41 & 162.91 \\
12 & 111.60 & 94.12 & 15.07 & 46.20 & 105.93 \\
13 & 111.09 & 121.62 & 127.00 & 45.69 & 181.68 \\
14 & 105.46 & -51.19 & -11.85 & 40.06 & 66.07 \\
\hline & 99.26 & -38.66 & -42.06 & 33.86 & 66.41 \\
\hline
\end{tabular}

* : $\mathrm{V}_{Z}=\mathrm{V}_{L S R}-\mathrm{V}_{\text {System }}$ where $\mathrm{V}_{\text {System }}$ is the system velocity of $65.4 \mathrm{~km} / \mathrm{s}$ as same the LSR velocity we used.

\section{DISCUSSIONS}

\subsection{Three Dimensional Kinematics of W51 Main}

Observational evidence of the existence of outflows powered by young stars is clear. Collimated outflows occur in most accretion system, through interaction of rotation and magnetic fields. These systems have various available tracers, such as masers, which can be 
Table 2 (Continued).

Space motion measurements from 2003 to 2006

\begin{tabular}{|c|c|c|c|c|c|}
\hline ID & $\mathrm{V}_{L S R}[\mathrm{~km} / \mathrm{s}]$ & $\mathrm{V}_{X}[\mathrm{~km} / \mathrm{s}]$ & $\mathrm{V}_{Y}[\mathrm{~km} / \mathrm{s}]$ & $\mathrm{V}_{Z}{ }^{*}[\mathrm{~km} / \mathrm{s}]$ & $\mathrm{V}_{S}[\mathrm{~km} / \mathrm{s}]$ \\
\hline 15 & 83.98 & -4.09 & -47.38 & 18.58 & 51.05 \\
\hline 16 & 82.61 & 5.11 & -0.83 & 17.21 & 17.98 \\
\hline 17 & 73.23 & -131.61 & 114.93 & 7.83 & 174.90 \\
\hline 18 & 83.21 & 1.63 & 106.57 & 17.81 & 108.06 \\
\hline 19 & 65.86 & 50.29 & -154.36 & 0.46 & 162.35 \\
\hline 20 & 55.46 & 37.44 & 99.00 & -9.94 & 106.30 \\
\hline $21^{R}$ & 124.45 & 1.85 & 2.74 & 59.05 & 59.15 \\
\hline 22 & 105.59 & -55.30 & -163.77 & 40.19 & 177.46 \\
\hline 23 & 104.96 & 155.29 & -101.45 & 39.56 & 189.66 \\
\hline 24 & 82.00 & 42.23 & 171.08 & 16.60 & 176.99 \\
\hline 25 & 63.51 & 99.18 & 140.30 & -1.89 & 171.83 \\
\hline 26 & 77.69 & -40.43 & -67.32 & 12.29 & 79.48 \\
\hline 27 & 74.28 & 6.46 & -15.21 & 8.88 & 18.75 \\
\hline 28 & 71.33 & 31.00 & 36.89 & 5.93 & 48.55 \\
\hline 29 & 69.53 & -1.05 & 3.88 & 4.13 & 5.76 \\
\hline 30 & 67.43 & -2.50 & -3.77 & 2.03 & 4.95 \\
\hline 31 & 66.21 & 1.12 & 2.75 & 0.81 & 3.07 \\
\hline 32 & 67.19 & 28.38 & 20.89 & 1.79 & 35.28 \\
\hline 33 & 60.22 & -16.36 & -17.87 & -5.18 & 24.78 \\
\hline 34 & 57.39 & -21.38 & -18.97 & -8.01 & 29.69 \\
\hline 35 & 55.91 & -19.72 & -36.52 & -9.49 & 42.58 \\
\hline 36 & 55.70 & 31.18 & 15.14 & -9.70 & 36.00 \\
\hline 37 & 55.56 & -22.92 & -37.49 & -9.84 & 45.03 \\
\hline 38 & 55.68 & -28.00 & -40.50 & -9.72 & 50.19 \\
\hline 39 & 73.29 & 33.57 & -83.69 & 7.89 & 90.52 \\
\hline 40 & 68.38 & -11.07 & 29.55 & 2.98 & 31.69 \\
\hline 41 & 73.86 & 48.32 & 26.91 & 8.46 & 55.95 \\
\hline 42 & 67.89 & -12.73 & -12.44 & 2.49 & 17.98 \\
\hline 43 & 68.95 & -11.45 & 64.46 & 3.55 & 65.57 \\
\hline 44 & 56.91 & -0.62 & 27.49 & -8.49 & 28.78 \\
\hline 45 & 51.48 & -37.11 & -37.69 & -13.92 & 54.69 \\
\hline 46 & 111.06 & -94.16 & 88.68 & 45.66 & 137.17 \\
\hline 47 & 7.33 & 1.85 & 2.84 & -58.07 & 58.17 \\
\hline 48 & 96.68 & 57.01 & 32.39 & 31.28 & 72.64 \\
\hline 49 & 88.53 & -100.70 & -89.66 & 23.13 & 136.80 \\
\hline 50 & 79.83 & 38.15 & 200.16 & 14.43 & 204.27 \\
\hline 51 & 71.73 & -34.74 & -45.04 & 6.33 & 57.24 \\
\hline 52 & 60.07 & -18.91 & -38.47 & -5.33 & 43.20 \\
\hline 53 & 57.84 & -28.32 & -81.28 & -7.56 & 86.40 \\
\hline 54 & 56.60 & -26.18 & -128.38 & -8.80 & 131.32 \\
\hline 55 & 55.88 & -43.80 & -75.58 & -9.52 & 87.87 \\
\hline 56 & 50.05 & -16.20 & -65.35 & -15.35 & 69.06 \\
\hline 57 & 113.76 & -74.14 & -82.53 & 48.36 & 121.02 \\
\hline 58 & 107.35 & 25.55 & -1.95 & 41.95 & 49.16 \\
\hline 59 & 107.74 & -27.72 & 33.85 & 42.34 & 60.89 \\
\hline 60 & 98.05 & -114.83 & 67.87 & 32.65 & 137.33 \\
\hline 61 & 106.31 & -30.80 & 40.24 & 40.91 & 65.13 \\
\hline 62 & 82.58 & -2.74 & 18.10 & 17.18 & 25.10 \\
\hline
\end{tabular}

* : $\mathrm{V}_{Z}=\mathrm{V}_{L S R}-\mathrm{V}_{\text {System }}$ where $\mathrm{V}_{\text {System }}$ is the system velocity of $65.4 \mathrm{~km} / \mathrm{s}$ as same the LSR velocity we used. $\mathrm{R}$ : Reference maser source 

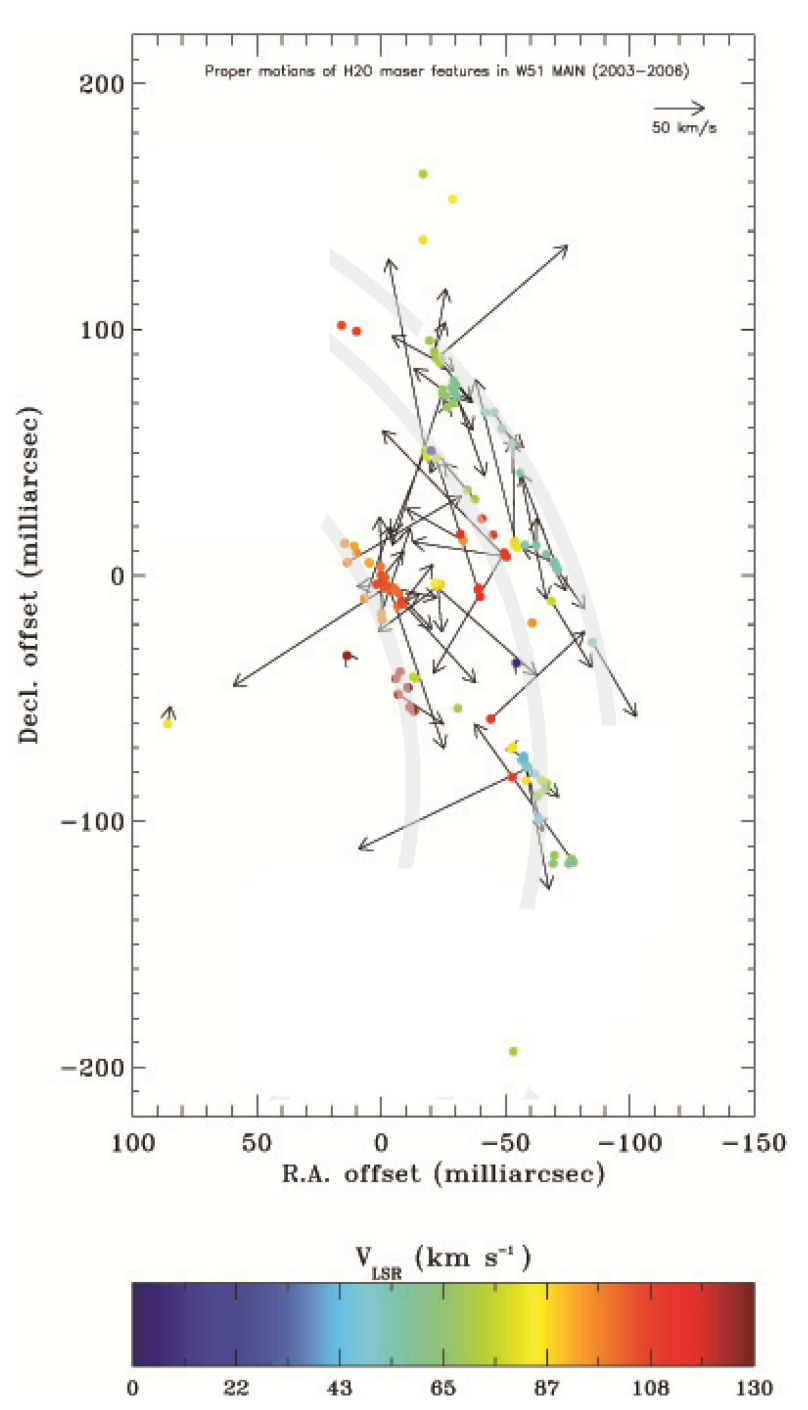

Fig. 5.- Proper motion vectors of $\mathrm{H}_{2} \mathrm{O}$ maser features from 2003 to 2006 with distributions of spots at 2006/031 in W51 Main. The arrow length of 20 mas represents velocities of $50 \mathrm{~km} / \mathrm{s}$ in the map centered on reference maser $(R)$ of data in 2006 [Subtracted velocity bias $(-1.821,-2.698) \mathrm{km} / \mathrm{s}]$.

investigate by proper motions and radial velocities as well as the distribution of spots.

We could find outflow motions of $\mathrm{H}_{2} \mathrm{O}$ masers excited by shocks in W51 Main from the internal motions as illustrated in Fig. 5. The velocities of proper motions have the range from $3 \mathrm{~km} / \mathrm{s}$ to $190 \mathrm{~km} / \mathrm{s}$ with a mean value of about $79 \mathrm{~km} / \mathrm{s}$ (Table 1). Fig. 6 shows the three-dimensional proper motions by using only blue-shifted (blue-color cones having negative values of $\mathrm{V}_{z}$ ) and red-shifted features (red-color cones having positive values of $\mathrm{V}_{z}$ ) in the radial direction based on Table 2, which summarizes spacial motion measurements of $\mathrm{H}_{2} \mathrm{O}$ masers in W51 Main. The range of the

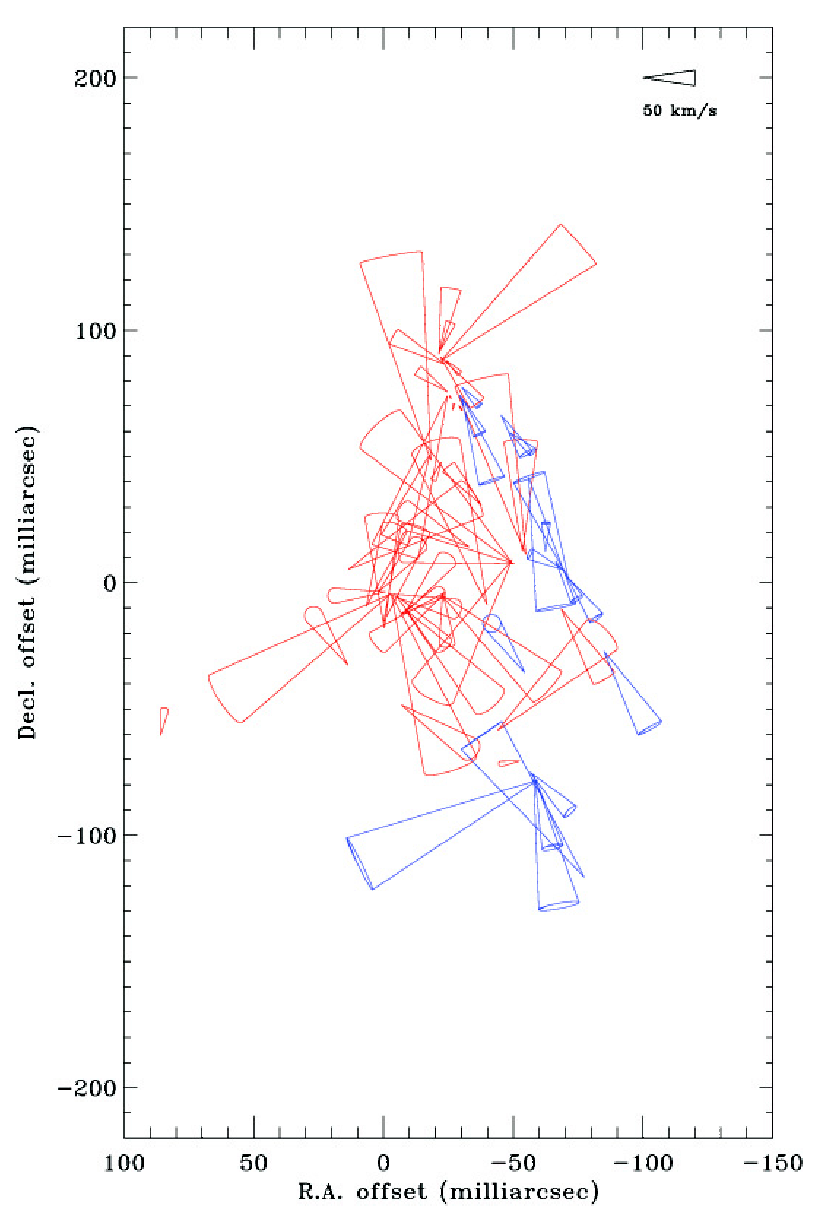

Fig. 6. - The three-dimensional motions of $\mathrm{H}_{2} \mathrm{O}$ maser features in W51 Main. Blue cones and red cones represent negative and positive values of $\mathrm{V}_{z}$, respectively. The cone length of 20 mas represents the space velocity of $50 \mathrm{~km} / \mathrm{s}$.

velocities is from $3 \mathrm{~km} / \mathrm{s}$ to $205 \mathrm{~km} / \mathrm{s}$, and has an average velocity of about $85 \mathrm{~km} / \mathrm{s}$. The random motions in W51 Main appear to be quite large compared to general maser proper motions as reported in Fish \& Reid (2007). Similar finding have previously been made for W49 North (Gwinn 1994) and also for our target W51 Main (Genzel et al. 1981; Imai et al. 2002).

We find no complete tracers of outflows probably because each tracer has its unique and limited physical condition itself. For perfect picture of outflows, it has been argued that observations in the multiwavelengths, such as X-ray, UV, optical, infrared, and radio, are required (Bally et al. 2007). Moreover, outflows have abilities to change the chemical composition of their host clouds and to force to reduce infall velocity (Arce et al. 2007). The high-resolution observations in multi-wavelengths seem to be critical to the detailed mapping of the kinematics of outflows from massive proto-stars. 


\subsection{Evolutionary Phase and Outflows}

UCH II regions represent young detectable stages in massive star formation. They are small, dense, and bright radio sources still embedded in their natal cloud (Churchwell 2002). Ionization of the natal material from $\mathrm{H}_{2}$ to $\mathrm{H}$ II needs a flux of Lyman continuum photons, $\mathrm{h} \nu>13.6 \mathrm{eV}$, from early-type stars. Many of UCH II regions have been detected to be associated with hot molecular cores (HMCs) (Krutz et al. 2000). Pascucci et al. (2004) has discussed the between HMCs and UCH II regions, and Kim \& Koo (2001) proposed a simple model, based on so-called champagne model, in which the supersonic gas flow drives a strong isothermal shock moving away from the core in contrast with the bow-shock model, and the hierarchical structure of massive star-forming regions for the extended emission around UCH II regions with HMCs. Their model and its conditions for $\mathrm{HCH}$ II region would be similar to environments of our target source, W51 Main. In this Kim \& Koo model, they considered that a massive star is formed at an off-center position within a hot core with $\mathrm{n}_{H_{2}} \sim 10^{7} \mathrm{~cm}^{-3}$ and $\sim 100 \mathrm{M}_{\odot}$, which is in a molecular clump of $\mathrm{n}_{\mathrm{H}_{2}} \sim 10^{5} \mathrm{~cm}^{-3}$. Hot cores, in general, have diameters $<0.1 \mathrm{pc}$, masses of $\mathrm{M} \sim$ $10^{2} \mathrm{M}_{\odot}$, densities greater than $\sim 10^{7} \mathrm{~cm}^{-3}$, and temperatures $>100 \mathrm{~K}$ (Kurtz et al. 2000; Kurtz 2000). They are regarded as potential precursors of the $\mathrm{HCH}$ II phases having diameter $<0.01$ pc (Kurtz 2000), and $\mathrm{HCH}$ II is thought to be precursors of the UCH II regions and the first indications of massive star formation with most massive clumps (Purcell et al. 2009). Hot cores are usually spatially offset by a few arcseconds from the UCH II regions, and always exist in the head of cometary UCH II regions (Cesaroni et al. 1994; Hofner et al. 1994). From these conditions similar to our target source having the averaged molecular hydrogen density $\sim 10^{5} \mathrm{~cm}^{-3}$ (Rudolph et al. 1990), scale $\sim 0.01 \mathrm{pc}$, and environments as shown in Fig. 3, we propose that W51 Main is around HCH II region and a massive star is under formation triggered by the pressure of the expanding UCH II region and the subsequential compression of the molecular gas like in the case of W3 region (Tieftrunk et al. 1997). Its morphology is apparently influenced by turbulent expansion of ionized gases from the massive star in UCH II region, W51 e2. Eventually, W51 Main will become the UCH II region with more complex structures.

Beuther \& Shepherd (2005) proposed possible evolutionary sequences of outflow by their observable outflow morphologies (Fig. 7). According to this scenario, W51 Main would be in the phase of the transition from the HCH II phase to the UCH II phase with embedded dust cocoon as in the models by Elmegreen \& Lada (1977) and Kim \& Koo (2001). W51 Main also exhibits environments and conditions outlined in Kurtz (2005) for HCH II region; i.e. (1) broader line profiles than general UCH II regions and (2) coincidence with the water maser position near UCH II region. Be-

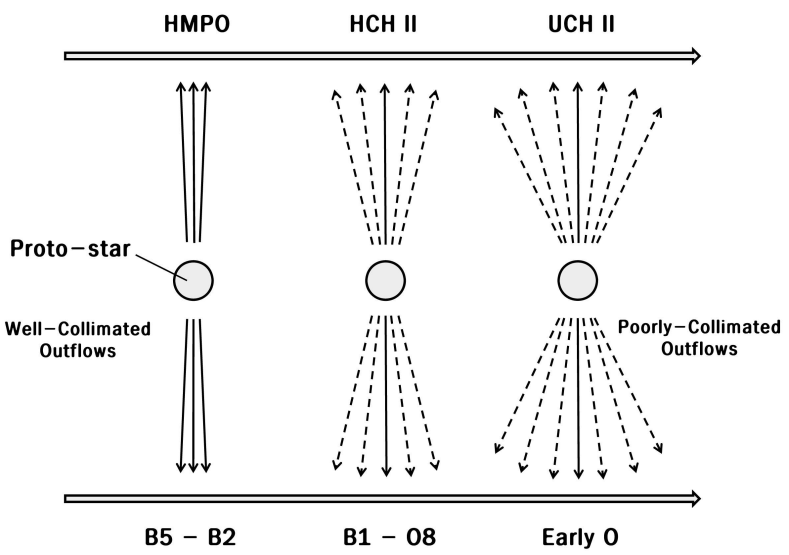

Fig. 7.- Sketch of proposed evolutionary sequences from high-mass proto-stellar object (HMPO) via $\mathrm{HCH}$ II to UCH II (upper arrow) with spectral type stages from B5-B2 via B1-O8 to early-O (bottom arrow) of massive proto-star by Beuther \& Shepherd (2005). (cf. Shepherd 2005, and Arce et al. 2007).

sides, the spectral type stage of massive proto-stars in W51 Main region would be earlier than B1, which is consistent with the result of Martin (1972); i.e. more than one $\mathrm{O}$ type star, from morphology of outflows, no well-collimated outflow features. These proto-stars in this highly massive star-forming region will become main-sequence stars in UCH II region. Moreover, we expect that this UCH II region will also make another shock layer(s) with the chain(s) of massive star forming regions.

\subsection{Speculations for W51 Main}

In W51 Main, we could easily identify structures which look like shell or filamentary threads (Fig. 5). These structures may have been resulted from stellar wind by newly-formed stars in nearby UCH II region. There is also a possibility that the structure is due to external stellar winds impacting the cloud. If this is the case, the structures would be parts of a larger structure with cometary morphology, or the structure may be affected by global magnetic field threading through the cloud (Yu et al. 1999). It is also possible that stringlike groups of spots could be elements in the same shell thread or the large shell structure, as considered in previous studies from correlation power spectra (Genzel \& Downes 1977; Genzel et al. 1979 \& 1981; Imai et al. 2002). Besides the stellar wind pressure, we could also suppose that there may be multiple originating points in a shell (Martin 1972; Imai et al. 2002). Magnetic and supersonic turbulences from newly-formed stellar object near our target may create such structure through fragmentation into multiple proto-stars (Zhang et al. 2009). Furthermore, we may also consider effects by poorly collimated outflow jets as described in section 4.2 with Fig. 7, or perhaps circum-stellar disk with accretion process. 
Finally, we caution that we always have to keep in mind the possibility of non-kinematic effects. That is, the observed proper motions may not be caused by direct motions of matters but due to random coherence in a turbulent medium or traveling excitation waves. In the latter case, motions of masers do not correlate with motions of matters (Fish \& Reid 2007).

\section{CONCLUSIONS AND FUTURE PROSPECTS}

We made maps of $\mathrm{H}_{2} \mathrm{O}$ maser spots in W51 Main, and measured three-dimensional motions of the spots from the multi-epoch observational data with VERA from 2003 to 2006 . From these results, we have constrained the evolutionary sequence of W51 Main and found that W51 Main contains multiple proto-stars. The environments and the position of W51 Main agree with the picture of a protostar with the compact H II region. We expect that proto-stars embedded in W51 Main region would be in an evolutionary phase in which $\mathrm{HCH}$ II regions are associated, and that the proto-stars would be evolved to stars with late-O spectral type.

The present study has a certain limitation in the sense that we base our analysis on the projected and relative distribution map without absolute positions of maser features. The map of $\mathrm{H}_{2} \mathrm{O}$ masers from phasereference technique is needed to measure absolute positions of maser features in the region.

\section{ACKNOWLEDGMENTS}

Cho was in part supported by the BK21 program of the Korean Government for Yonsei University. Kan-Ya acknowledges the support of the same BK21 program. Byun was supported by National Research Foundation of Korea through Grant 2009-0075376.

\section{REFERENCES}

Arce, H. G., Shepherd, D., Gueth, F., Lee, C.-F., Bachiller, R., Rosen, A., \& Beuther, H. 2007, Molecular Outflows in Low- and High-Mass Star-Forming Regions, in Protostars and Planets V, ed. Reipurth B., Jewitt D., Keil K. (Tucson: University of Arizona Press), 245

Argon, A. L., Reid M. J., \& Menten, K. M. 2002, FullPolarization Maps of OH Main-Line Masers in the W51 and G351.78-0.54 Star-Forming Regions, IAU Symp., 206, 367

Bally, J., Reipurth, B., \& Davis, C. J. 2007, Observations of Jets and Outflows from Young Stars, in Protostars and Planets V, ed. Reipurth B., Jewitt D., Keil K. (Tucson: University of Arizona Press), 215

Beuther, H., Churchwell, E. B., McKee, C. F., \& Tan, J. C. 2007, The Formation of Massive Stars, in Protostars and Planets V, ed. Reipurth B., Jewitt D., Keil K. (Tucson: University of Arizona Press), 165

Beuther, H., \& Shepherd, D. 2005, Precursors of UCHII Regions \& the Evolution of Massive Outflows, A\&SSL, 324,105
Cesaroni, R., Churchwell, E., Hofner, P., Walmsley, C. M., \& Krutz, S. 1994, Hot Ammonia towards Compact HII Regions, A\&A, 288, 903

Cheung, A. C., Rank, D. M., \& Townes, C. H. 1969, Detection of Water in Interstellar Regions by its Microwave Radiation, Nature, 221, 626

Chikada, Y., Kawaguchi, N., Inoue, M., Morimoto, M., Kobayashi, H., \& Mattori S. 1991, The VSOP Correlator, in Frontiers of VLBI, Tokyo: Universal Academy Press, 79

Churchwell, E. 2002, Ultra-Compact HII Regions and Massive Star Formation, ARA\&A, 40, 27

De Wit, W. J., Testi, L., Palla, F., \& Zinnecker, H. 2005, The Origin of Massive O-Type Field Stars: II. Field O Stars as Runaways, A\&A, 437, 247

Elmegreen, B. G., \& Lada, C. J. 1977, Sequential Formation of Subgroups in OB Associations, ApJ, 214, 725

Fish, V. L., \& Reid, M. J. 2007, Proper Motions of OH Masers and Magnetic Fields in Massive Star-Forming Regions, ApJ, 670, 1159

Fomalont, E. B., \& Weliachew, L. 1973, Interferometric Observations of Formaldehyde Absorption in Front of Strong Galactic Sources, ApJ, 181, 781

Genzel, R., \& Downes, D. 1977, $\mathrm{H}_{2} \mathrm{O}$ in the Galaxy: Sites of Newly Formed OB Stars, A\&AS, 30, 145

Genzel, R., Downes, D., Moran, J. M., Johnston, K. J., Spencer, J. H., Matveyenko, L. I., Kogan, L. R., Kostenko, V. I., Rönnäng, B., Haschick, A. D., Reid, M. J., Walker, R. C., Giuffrida, T. S., Burke, B. F., \& Moiseev, I. G. 1979, $\mathrm{H}_{2} \mathrm{O}$ in W51 Main - An Expanding Bubble around a Young Massive Star, A\&A, 78, 239

Genzel, R., Downes, D., Schneps, M. H., Reid, M. J., Moran, J. M., Kogan L. R., Kostenko, V. I., Matveyenko, L. I., \& Ronnang, B. 1981, Proper Motions and Distances of $\mathrm{H}_{2} \mathrm{O}$ Maser Sources. II - W51 MAIN, ApJ, 247, 1039

Gwinn, C. R. 1994, Hypersonic Acceleration and Turbulence of $\mathrm{H}_{2} \mathrm{O}$ Masers in W49N, ApJ, 429, 241

Goldader, J. D., \& Wynn-Williams, C. G. 1994, NearInfrared Observations of W51, ApJ, 433, 164

Hofner, P., Krutz S., Churchwell, E., Walmsley, C. M., \& Cesaroni, R. 1994, High-Resolution Observations of a New Ammonia Maser Line in G9.62+0.19, ApJ, 429, L85

Honma, M., \& the VERA collaboration 2003, First Fringe Detection with VERAs Dual-Beam System and Its PhaseReferencing Capability, PASJ, 55, L57

Honma, M., \& the VERA collaboration 2005, Multi-Epoch VERA Observations of -0.1, PASJ, 57, 595

Iguchi, S., Kurayama, T., Kawaguchi, N., \& Kawakami, K. 2005, Gigabit Digital Filter Bank: Digital Backend Subsystem in the VERA Data-Acquisition System, PASJ, 57,259

Imai, H., Watanabe, T., Omodaka, T., Nishio, M., Kameya, O., Miyaji, T., \& Nakajima, J. 2002, 3-D Kinematics of Water Masers in the W 51A Region, PASJ, 54, 741

Imai, H., \& the VERA collaboration 2005, Water masers in W51A observed with the VLBI Exploration of Radio 
Astrometry VERA, in Massive Star Birth : A Crossroads of Astrophysics, IAU Symp. 242, poster section

Imai, H. 2006, Observational Study on Star Formation with VLBI (Kiyosato: EAYAM 2006)

Jaffe, D. T., \& Martin-Pintdo. 1999, Low-Velocity Ionized Winds from Regions around Young O Stars, ApJ, 520, 162

Kan-ya, Y., \& the VERA collaboration 2007, CCcoda The I/O Library for the Radio Data Reduction System VEDA, BKAS, 32, No.1, 104

Kim, K.-T., \& Koo, B.-C. 2001, Radio Continuum and Recombination Line Study of Ultracompact H II Regions with Extended Envelopes, ApJ, 549, 979

Kurtz, S., Cesaroni, R., Churchwell, E., Hofner, P., \& Walmsley, C. M. 2000, Hot Molecular Cores and the Earliest Phases of High-Mass Star Formation, in Protostars and Planets IV, ed. Mannings V., Boss A., Russell S. S. (Tucson: University of Arizona Press), 299

Kurtz, S. 2000, Ultracompact H II Regions: New Challenges, Rev. Mex. AA, 9, 169

Krutz, S. 2005, Hypercompact H II Regions, in Massive Star Birth, IAU Symp. 227, 111

Lai, S.-P., Crutcher, R. M., Girart, J. M., \& Rao, R. 2001, Interferometric Mapping of Magnetic Fields in Star-Forming Regions. I. W51 e1/e2 Molecular Cores, ApJ, 561, 864

Leppänen, K., Liljeström, T., \& Diamond, P. 1998, Submilliarcsecond Linear Polarization Observations of Water Masers in W51 M, ApJ, 507, 909

Liljeström, T. 1998, Shock Structure of the High-Velocity Water Masers in W49 N, ASPCS, 144, 251

Martin, A. H. M. 1972, High Resolution Observations of W51, MNRAS, 157, 31

Pascucci, I., Apai, D., Henning, Th., Stecklum, B., \& Brandl, B. 2004, The Hot Core-Ultracompact H II Connection in G10.47+0.03, A\&A, 426, 523

Purcell, C. R., Longmore, S. N., Burton, M. G., Walsh, A. J., Minier, V., Cunningham, M. R., \& Balasubramanyam, R. 2009, Physical and Chemical Conditions in Methanol Maser Selected Hot Cores and UCHII Regions, MNRAS, 394, 323

Reid, M. J. 2002, Masers from Protostars to Black Holes: Conference Summary, ASPCS, 206, 506

Rudolph, A., Welch, W. J., Palmer, P., \& Dubrulle, B. 1990, Dynamical Collapse of the W51 Star-Forming Region, ApJ, 363, 528

Sato, M., \& the VERA collaboration 2007, Absolute Proper Motions of $\mathrm{H}_{2} \mathrm{O}$ Masers away from the Galactic Plane Measured with VERA in the Superbubble Region NGC 281, PASJ, 59, 743

Scott, P. F. 1978, High Resolution Radio Observations of G49.5-0.4 W51, MNRAS, 183, 435

Sewilo, M., Chuchwell, E., Krutz, S., Goss, W. M., \& Hofner, P. 2004, Broad Radio Recombination Lines from Hypercompact H II Regions, ApJ, 605, 285

Shepherd, D. 2005, Massive Star Outflows, in Massive Star Birth, IAU Symp., 227, 237
Tieftrunk, A. R., Gaume, R. A., Cluassen, M. J., Wilson, T. L., \& Johnston, K. J. 1997, The H II Molecular Cloud Complex W3 Revisited: Imaging the Radio Continuum Sources Using Multi-Con, Multi-Frequency Observations with the VLA, A\&A, 318, 931

Torrelles, J. M., Gomez, J. F., Rodriguez, L. F., Curiel, S., Ho, P. T. P., \& Garay, G. 1996, The Thermal Radio Jet of Cepheus A HW2 and the Water Maser Distribution at 0 -8pt. Scale (60 AU), ApJ, 457, L107

Ulich, B. L., \& Has,s R. W. 1976, Absolute Calibration of Millimeter-Wavelength Spectral Lines, ApJS, 30, 247

Wood, D. O. S., \& Churchwell, E. 1989, The Morphologies and Physical Properties of Ultracompact H II Regions, ApJS, 69, 831

Yu, K. C., Billawala, Y., \& Bally, J. 1999, Parsec-Scale CO Outflow and $\mathrm{H}_{2}$ Jets in Barnard 5, ApJ, 118, 2940

Zhang, Q., \& Ho, P. T. P. 1997, Dynamical Collapse in W51 Massive Cores: $\mathrm{NH}_{3}$ Observations, ApJ, 488, 241

Zhang, Q., Wang, Y., Pillai, T., \& Rathborne, J. 2009, Fragmentation at the Earliest Phase of Massive Star Formation, ApJ, 696, 268

Zinnecker, H., \& Yorke, H. W. 2007, Toward Understanding Massive Star Formation, ARA\&A, 45, 481 


\section{APPENDIX A. Measurement of Relative Proper Motions of $\mathrm{H}_{2} \mathrm{O}$ Masers}

Fig. 8, 9 and 10 show all cases of measured relative proper motions of $\mathrm{H}_{2} \mathrm{O}$ maser spots with ID as given in Table 1 . Constant velocity is assumed for drawing fitted lines (Imai et al. 2002). Some features have large offset values relative to other epochs or fit lines because of several possibilities, such as poor-fit values relative to the origin, the errors within the size of angular resolution (1.2 mas), poor-calibrated data, and weather conditions, and so on. However, we contained all offset spots within criteria because they do not significantly affect whole systematic motions in W51 Main.

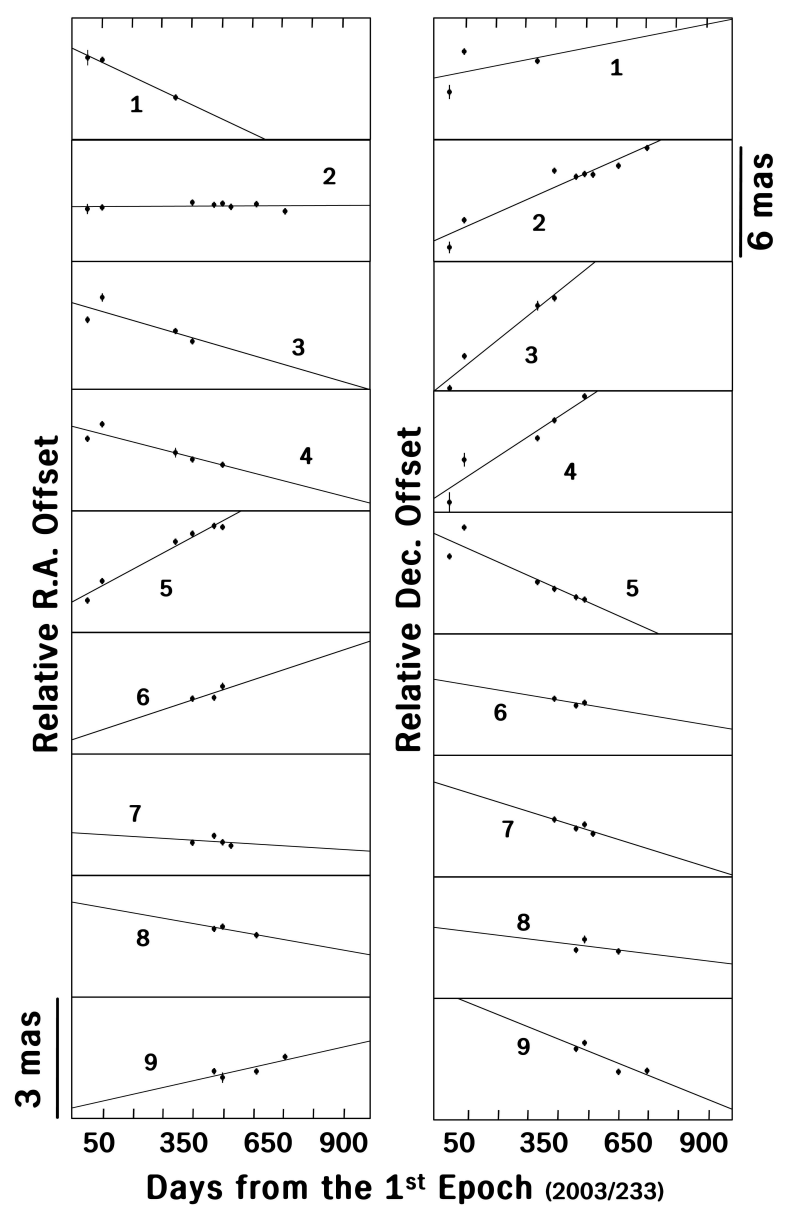

Fig. 8.- Observed relative proper motions of all $\mathrm{H}_{2} \mathrm{O}$ maser features (ID numbers from 1 to 24) in W51 Main. The number marked within each box represents the ID in Table 1. The solid line represents linear least-square-fitted line assuming a constant velocity motion. Each diagram has same height corresponding to 3 mas except diagrams with additional right-side notes.

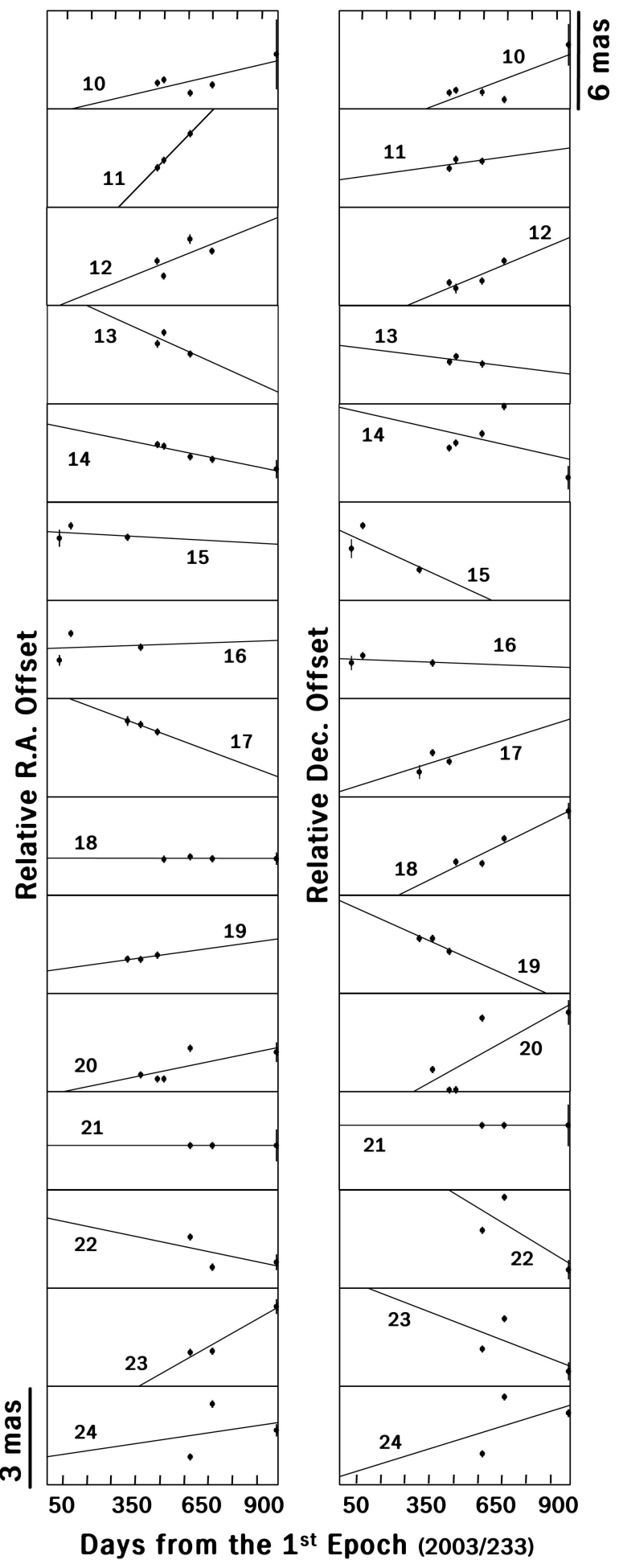



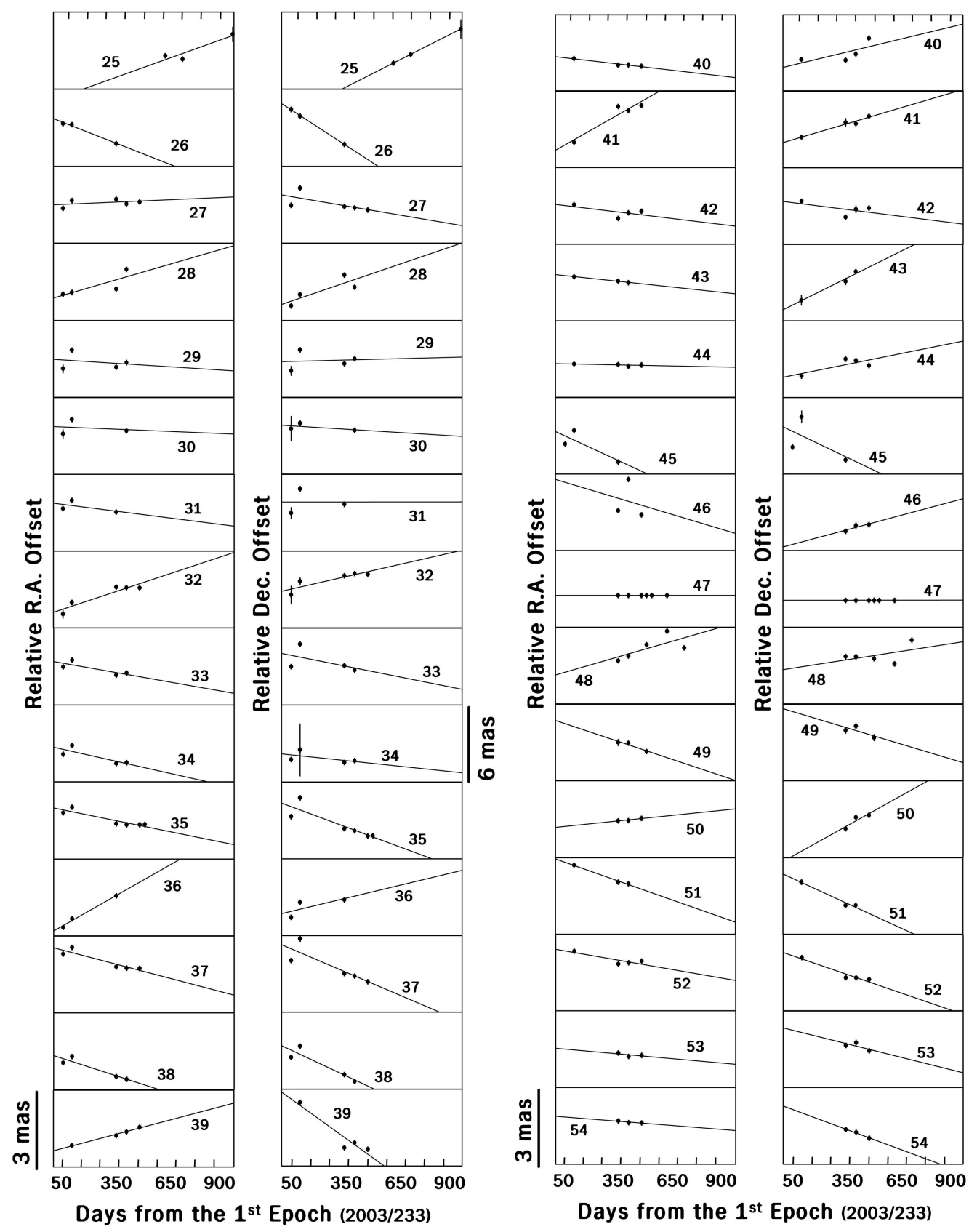

Fig. 9.- Observed relative proper motions of all $\mathrm{H}_{2} \mathrm{O}$ maser features (continued ID numbers from 25 to 54 ) in W51 Main. 


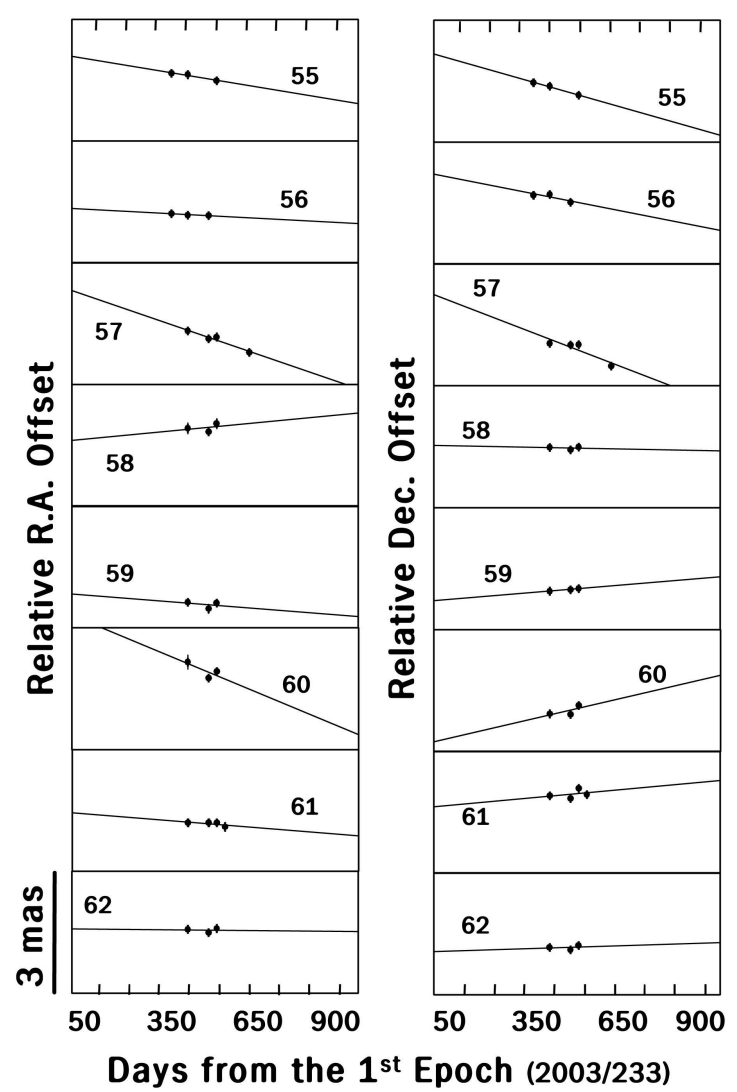

Fig. 10.- Observed relative proper motions of all $\mathrm{H}_{2} \mathrm{O}$ maser features (continued ID numbers from 55 to 62 ) in W51 Main. 